\title{
Klinische Anwendung der Computertomographie beim Rind
}

\author{
Nuss, Karl ; Schnetzler, C ; Hagen, Regine ; Schwarz, Andrea ; Kircher, Patrick R
}

\begin{abstract}
Die Computertomographie liefert mithilfe von Röntgenstrahlen Schnittbilder von Körperregionen. Diese zeigen eine überlagerungsfreie, zweidimensionale Darstellung aller gewünschten Ebenen und ermöglichen auch eine dreidimensionale Rekonstruktion interessierender Körperregionen. Berichte über die Computertomographie beim Rind finden sich nur vereinzelt. Hohe Kosten, medikamentöse Restriktionen und die notwendige Allgemeinanästhesie limitieren die Anwendungen. Indikationen beim Rind sind vor allem Erkrankungen des Kopfes - wie Zahnerkrankungen oder Otitis media - sowie neurologische Erkrankungen, weniger oft Erkrankungen der Wirbelsäule und Gliedmaßen. Bei wertvollen Rindern, bei denen eine operative Therapie geplant ist, kann die Computertomographie Befunde liefern, die für die Durchführung des Eingriffs entscheidend sind. Die computertomographischen Befunde können andererseits auch dazu beitragen, eine ungünstige Prognose zu erkennen und so unnötige Operationen zu vermeiden. Abstract: Computed tomography involves the use of x-rays to produce cross-sectional images of body regions. It provides non-overlapping, two-dimensional images of all desired planes as well as three-dimensional reconstruction of regions of interest. There are few reports on the clinical use of computed tomography in farm animals. Its use in cattle is limited by high cost, the application of off-label drugs and the need for general anaesthesia. In cattle computed tomography is indicated primarily for diseases of the head, e.g. dental diseases and otitis media, and neurological disorders. Less often it is used for diseases of the vertebrae and limbs. In valuable cattle, the results of computed tomography can be an important part of preoperative planning or be used to avoid unnecessary surgery when the prognosis is poor.
\end{abstract}

DOI: https://doi.org/10.1055/s-0038-1623076

Other titles: Clinical application of computed tomography in cattle

Posted at the Zurich Open Repository and Archive, University of Zurich

ZORA URL: https://doi.org/10.5167/uzh-50114

Journal Article

Published Version

Originally published at:

Nuss, Karl; Schnetzler, C; Hagen, Regine; Schwarz, Andrea; Kircher, Patrick R (2011). Klinische Anwendung der Computertomographie beim Rind. Tierärztliche Praxis. Ausgabe G, Grosstiere/Nutztiere, 39(5):317-324.

DOI: https://doi.org/10.1055/s-0038-1623076 


\title{
Klinische Anwendung der Computertomographie beim Rind
}

\author{
K. Nuss ${ }^{1}$; C. Schnetzler ${ }^{1}$; R. Hagen ${ }^{2}$; A. Schwarz ; P. Kircher $^{2}$
}

${ }^{1}$ Departement für Nutztiere, Vetsuisse-Fakultät der Universität Zürich, Schweiz; ${ }^{2}$ Abteilung Bildgebende Diagnostik, Departement für Kleintiere, Vetsuisse-Fakultät der Universität Zürich, Schweiz; ${ }^{3}$ Abteilung Anästhesiologie, Departement für Pferde, Vetsuisse-Fakultät der Universität Zürich, Schweiz

\section{Schlüsselwörter}

Rind, bildgebende Diagnostik, Computertomographie, Kopf, Gliedmaßen

\section{Zusammenfassung}

Die Computertomographie liefert mithilfe von Röntgenstrahlen Schnittbilder von Körperregionen. Diese zeigen eine überlagerungsfreie, zweidimensionale Darstellung aller gewünschten Ebenen und ermöglichen auch eine dreidimensionale Rekonstruktion interessierender Körperregionen. Berichte über die Computertomographie beim Rind finden sich nur vereinzelt. Hohe Kosten, medikamentöse Restriktionen und die notwendige Allgemeinanästhesie limitieren die Anwendungen. Indikationen beim Rind sind vor allem Erkrankungen des Kopfes - wie Zahnerkrankungen oder Otitis media - sowie neurologische Erkrankungen, weniger oft Erkrankungen der Wirbelsäule und Gliedmaßen. Bei wertvollen Rindern, bei denen eine operative Therapie geplant ist, kann die Computertomographie Befunde liefern, die für die Durchführung des Eingriffs entscheidend sind. Die computertomographischen Befunde können andererseits auch dazu beitragen, eine ungünstige Prognose zu erkennen und so unnötige Operationen zu vermeiden.

\section{Key words}

Cattle, diagnostic imaging, computed tomography, head, limbs

\section{Summary}

Computed tomography involves the use of $\mathrm{x}$-rays to produce cross-sectional images of body regions. It provides non-overlapping, two-dimensional images of all desired planes as well as three-dimensional reconstruction of regions of interest. There are few reports on the clinical use of computed tomography in farm animals. Its use in cattle is limited by high cost, the application of off-label drugs and the need for general anaesthesia. In cattle computed tomography is indicated primarily for diseases of the head, e. g. dental diseases and otitis media, and neurological disorders. Less often it is used for diseases of the vertebrae and limbs. In valuable cattle, the results of computed tomography can be an important part of preoperative planning or be used to avoid unnecessary surgery when the prognosis is poor.

\section{Korrespondenzadresse}

Karl Nuss

Prof. Dr. med. vet., Dipl. ECVS, Dipl. ECBHM

Departement für Nutztiere

Vetsuisse-Fakultät der Universität Zürich

Winterthurerstrasse 260

8057 Zürich, Schweiz

E-Mail:knuss@vetclinics.uzh.ch
Clinical application of computed tomography in cattle

Tierärztl Prax 2011; 39 (G): 317-324

Eingegangen: 18. Januar 2011

Akzeptiert nach Revision: 26. Juli 2011

\section{Einleitung}

In der Kleintier- und der Pferdechirurgie sowie -medizin hat sich die Computertomographie fest etabliert. In der Nutztierpraxis hingegen kommen bildgebende Verfahren aus ökonomischen Gründen generell weniger oft zur Anwendung als bei Kleintieren und Pferden $(15,28,29,34)$. Allenfalls die Ultraschall- und die Röntgenuntersuchung werden öfter vorgenommen. In der Literatur finden sich jedoch zunehmend Berichte über die computertomographische $(8,20,23,36-38)$ und sogar die magnetresonanztomographische $(36,40,41)$ Untersuchung beim Rind. Neben experimentellen Untersuchungen, die die Anatomie einzelner Körperabschnitte beschreiben (30), werden auch klinische Fallberichte oder -serien publiziert.

\section{Technische Grundlagen der Computertomographie}

Bei der Computertomographie handelt es sich um ein Schnittbildverfahren, das sich der Röntgenstrahlung bedient. Da die Technik viele der Grundlagen, beispielsweise die Erzeugung der Strahlen und deren Abschwächung durch die Gewebe, mit der Röntgendiagnostik gemeinsam hat (15), sind die Aufnahmen ähnlich wie ein Röntgenbild zu interpretieren. Das Gewebe der zu untersuchenden Körperregion wird durch eine in Längsrichtung um den Körper rotierende Röntgenröhre kontinuierlich aus verschiedenen Winkeln durchstrahlt. Der Röntgenstrahl breitet sich nicht in alle Richtungen aus, sondern lässt sich auf eine bestimmte Schichtdicke, meist zwischen 1 und $10 \mathrm{~mm}$, eingrenzen. Während der rotierenden, linearen Abtas- 
tung wird die räumliche Verteilung der Strahlenprofile nach dem Durchtritt durch den Patienten durch Detektorenreihen aufgezeichnet. Analog zur Röntgenuntersuchung hängt die Stärke der Strahlenabsorption von der Ordnungszahl der Atome und der Elektronendichte des Gewebes ab. Das heißt, ein einziger physikalischer $\mathrm{Pa}$ rameter, die Abschwächung der Röntgenstrahlen, bestimmt die spätere Darstellung des Gewebes auf dem Bild. Gewebe mit hoher Elektronendichte (z. B. Knochen) absorbieren die Strahlung stärker als Gewebe mit geringerer Dichte (z. B. Flüssigkeit oder Fett). Die durchschnittliche relative Strahlenabsorptionsrate kann nun pro Volumeneinheit - kurz als Voxel (engl. volumetric element) bezeichnet - berechnet werden (14). Die berechnete durchschnittliche Gewebeabsorption eines bestimmten Gewebes wird in HounsfieldEinheiten (Hounsfield Units, HU) angegeben und kann als Bildelement - Pixel (engl. picture element) genannt - in verschiedenen Graustufen zweidimensional sichtbar gemacht werden (16). Ein Pixel repräsentiert ein dreidimensionales Voxel im Patienten. Die so genannte Hounsfield-Skala rangiert zwischen -1000 für Luft bis +1000 für Knochen. Der HU-Wert 0 wurde dem Absorptionswert von Wasser zugeordnet $(24,32)$. Kontrastmittel können je nach Konzentration Werte von +3000 HU erreichen. Ein Computer wandelt die gesammelten Daten mit verschiedenen mathematischen Verfahren in ein Bild um. Die Nachbearbeitung am Computer erlaubt die Hervorhebung bestimmter Dichtestufen, mithin die „Fensterung“ interessierender Regionen (15).

Computertomographen können bis zu 4000 verschiedene Absorptionswerte differenzieren. Somit lassen sich ebenso viele Graustufen, sowie zusätzlich Schwarz und Weiß, abbilden. Das menschliche Auge vermag jedoch nur ca. 200 verschiedene Grau-

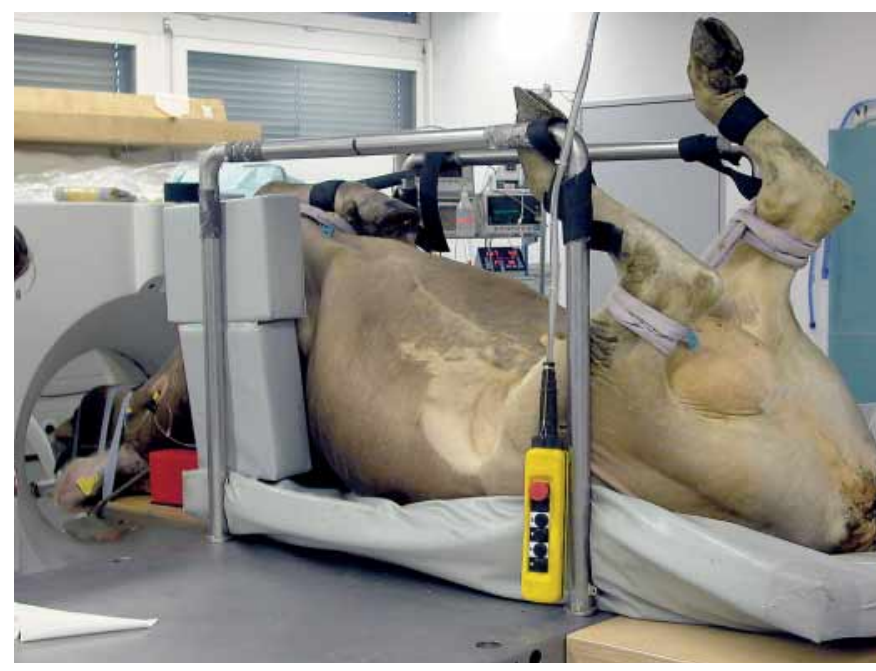

Abb. 1 Lagerung und Fixation einer Kuh in Rückenlage unter Allgemeinanästhesie zur computertomographischen Untersuchung des Schädels. Spezieller Tisch mit beweglicher Tischplatte, Kran zum Anheben und Transportieren der Kuh.

Fig. 1 A cow under general anaesthesia is positioned and secured in dorsal recumbency for computed tomographic evaluation of the skull. A specialized table with a movable tabletop and a crane to lift the cow were used. töne zu unterscheiden. Aus diesem Grund bedient man sich bei der Wiedergabe der Bilder der so genannten Fenstertechnik. Dabei wird nur ein bestimmter, für das menschliche Auge differenzierbarer Bereich der Hounsfieldskala dargestellt. Die „Fensterweite“ beschreibt dabei die Spannweite auf der Hounsfieldskala, über den die Grautöne verteilt werden. Ein „weich“ eingestelltes Fenster, das heißt ein Fenster mit einer kleinen HU-Spannweite, führt zu einer guten Kontrastauflösung innerhalb des Fensters. Die „Fensterlage“ stellt das Zentrum des Fensters dar und sollte dem Gewebe von Interesse entsprechen. Die Gewebe außerhalb des gewählten Fensters werden weiß (oberhalb) und schwarz (unterhalb) dargestellt.

Der wichtigste Vorteil der Computertomographie gegenüber dem konventionellen Röntgen ist die überlagerungsfreie Darstellung der interessierenden Körperregion. Dadurch gelingt es vergleichsweise einfacher als bei der Röntgenuntersuchung, die genaue anatomische Lokalisation, das Ausmaß der Veränderungen und mögliche operative Zugänge zu bestimmen. Zudem liefert die Computertomographie genauere Informationen über die Ausdehnung und das Volumen von Organen (17). Ferner können dreidimensionale Rekonstruktionen von Körperteilen berechnet und abgebildet werden, die es erlauben, insbesondere Knochenstrukturen wie anatomische Präparate darzustellen (4, 27). Mittels digitaler Bearbeitung ist es möglich, „Fenster“ in Knochen zu schneiden und so „in den Knochen hineinzusehen“, was sich vor allem im Bereich des Schädels und der Sinus als diagnostisch wertvoll erweist. Die örtliche Auflösung der Computertomographie ist erheblich geringer als die der konventionellen Röntgenbilddarstellung (32). Dagegen verfügt das CT-Bild über eine bessere Kontrastauflösung als die Röntgenaufnahme. Nachteile der Computer-

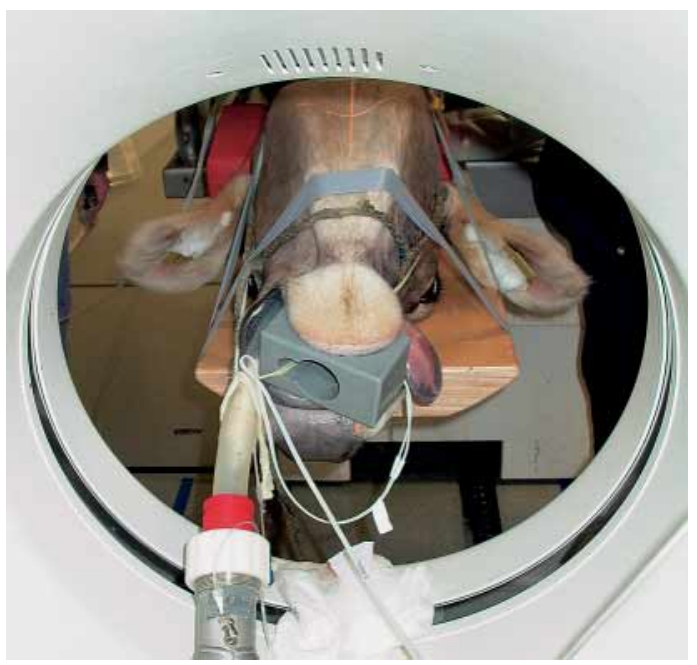

Abb. 2 Exakte Positionierung des Kopfes zur optimalen Darstellung des interessierenden Bereichs in der Gantry-Öffnung. Lichtkreuz zur Lokalisation des durchstrahlten Bereichs.

Fig. 2 Precise positioning of the head in the gantry opening for optimal visualisation of the region of interest. The crosshairs indicate the region to be x-rayed. 
tomographie sind hohe Kosten, die stark erhöhte Strahlenbelastung für den Patienten, die limitierte Aussagekraft bei Weichteilen und die Entstehung von Artefakten in gewissen Regionen, z. B. im Bereich der Medulla oblongata (15).

\section{Vorbereitung des Patienten}

Die korrekte Lagerung des Patienten und des gewünschten Untersuchungsbereichs stellt eine unerlässliche Voraussetzung für eine gute Bildqualität dar. Dies beinhaltet die anatomische Symmetrie, die zentrale Positionierung der zu untersuchenden Region in der Gantry und die Entfernung aller störenden Gegenstände oder Körperteile, da diese die Bildqualität beeinträchtigen oder Artefakte erzeugen können.
Der Einsatz der Computertomographie beim adulten Rind erfordert eine spezielle Tischkonstruktion ( $>$ Abb. 1) (34). Der Durchmesser der Gantry-Öffnung ( $\$$ Abb. 2) begrenzt die Nutzung der Computertomographie bei Großtieren auf die Untersuchung des Schädels, des kranialen Teils der Halswirbelsäule und der Gliedmaßen (1). Beim Rind wurden bisher oftmals Präparate untersucht $(18,26,31,33)$, die vor allem dem Vergleich der Anatomie mit der bildgebenden Darstellung dienten. Zur Vermeidung von Bewegungsartefakten sowie von Schäden am Patienten und Computertomographen ist eine Allgemeinnnarkose der Tiere notwendig (23). Dieser erhöhte Aufwand schränkt die Anwendung bei Nutztieren weiter ein. Die neuesten Generationen der Computertomographen ermöglichen jedoch meist eine Reduktion der Untersuchungszeit auf wenige Minuten, sodass durchschnittlich eine Allgemeinanästhesie von 15-30 Minuten ausreicht (19, 20).
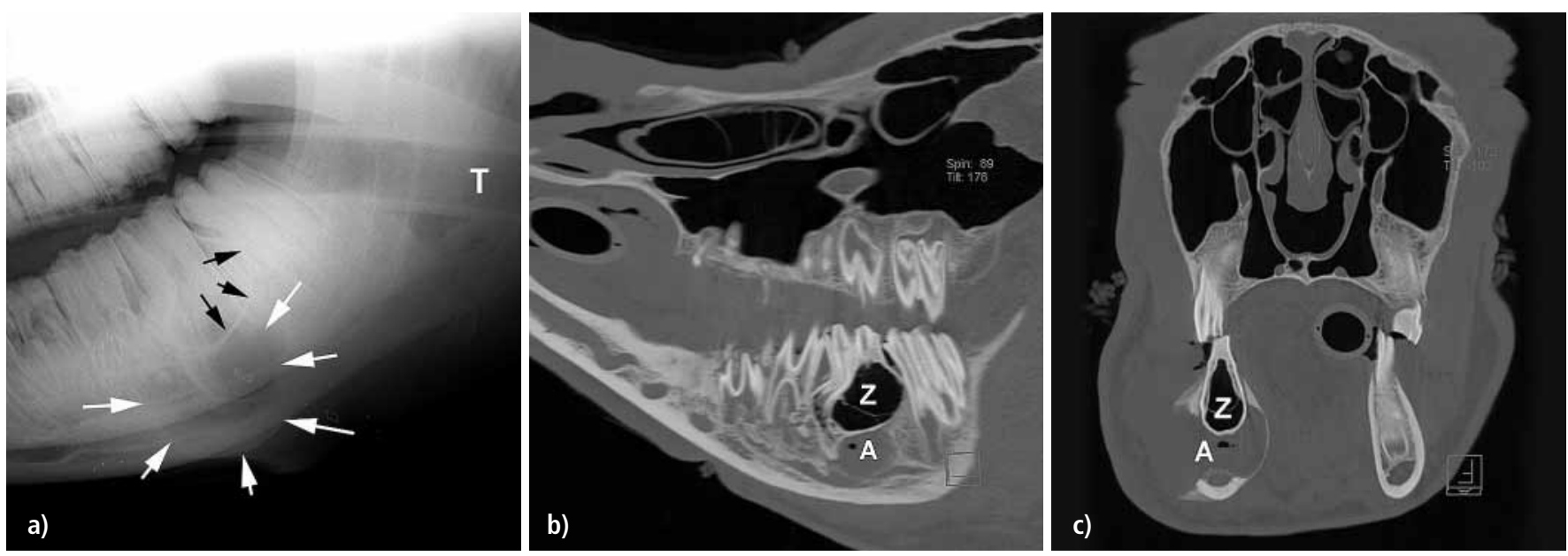

Abb. 3 Jungrind, 2 Jahre, Umfangsvermehrung am Unterkiefer. a) Röntgenbild des Unterkiefers im laterolateralen Strahlengang unter Allgemeinanästhesie: große lytische Zone (weiße Pfeile) im Bereich des M2, kaudale Zahnwurzel nicht sichtbar, fast horizontal verlaufende Linie (schwarze Pfeile). Metallclips kennzeichnen den Verlauf der A. und V. facialis. T = Tracheotubus. b) Longitudinales Schnittbild durch den betroffenen M2: große luftgefüllte Zyste (Z) in der kaudalen Wurzel; Verdrängung und Deformation der kranialen Wurzel sowie der benachbarten Zähne. Weichteildichte runde Zubildung mit Gaseinschluss ventral der abnorm geformten Zahnwurzel kennzeichnet den Wurzelabszess (A). c) Transversales Schnittbild durch den betroffenen M2: Verdeutlichung der Ausdehnung von Zyste (Z) und Wurzelabszess (A). d) Dreidimensional wirkende Rekonstruktion des Schädels. Auftreibung im Unterkieferknochen mit großem osteolytischem Bereich, in dem die kraniale Wurzel sichtbar und die Zyste kaudal abgrenzbar (gepunktete Linie) ist; komplette Perforation des Unterkieferknochens; Fehlstellung von M3 und $\mathrm{M} 1$ aufgrund der Verdrängung durch die Zyste.

Fig. 3 a) Laterolateral radiograph of a mandibular enlargement in a 2-year-old heifer under general anaesthesia: large lytic area (white arrows) near the second molar, of which the caudal root is not visible. The black arrows indicate a line running dorsal to the lytic area. The course of the facial artery and vein is marked by metal clips. $T$ = endotracheal tube. b) Longitudinal computed tomographic image of the diseased second molar: large air-filled cyst (Z) in the caudal root and displacement and deformation of the cranial root and adjacent teeth. The round soft tissue density with a gas-inclusion ventral to the deformed tooth root is a tooth root abscess (A). c) Transverse computed tomographic image of the diseased second molar. The size of the cyst (Z) and tooth root abscess (A) are clearly visible. d) Computed tomographic re-construction with 3D effect of the skull. The mandibular enlargement with a large lytic area in which the cranial root is visible and the delineated cyst (broken line) are shown. There is complete perforation of the mandible, and displacement of the first and third molars by the cyst. 


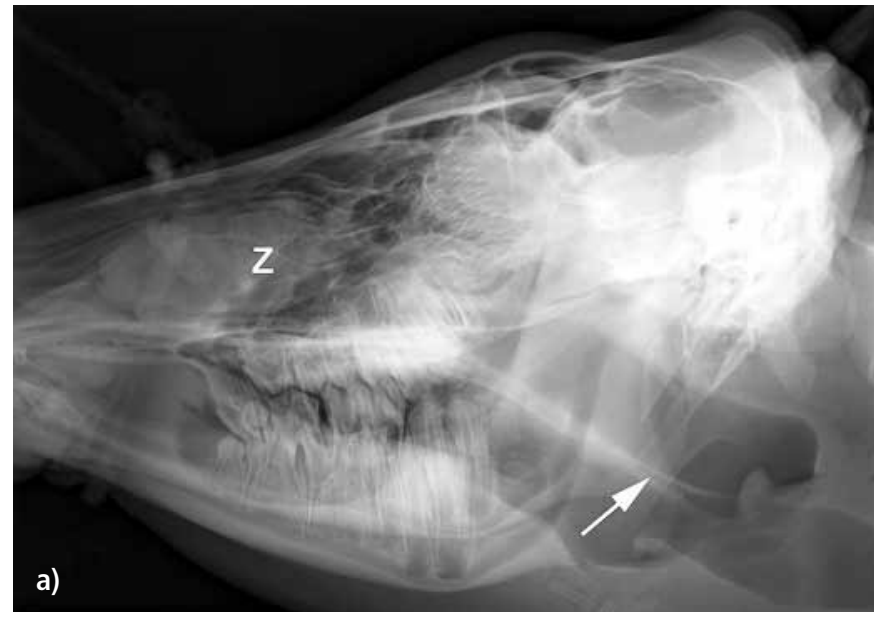

Abb. 4 a) Conchonasale Zyste bei einem Jungrind mit in- und exspiratorischem Atemgeräusch, Röntgenaufnahme im laterolateralen Strahlengang: weichteildichte Masse (Z) mit knochendichter Wandung im Bereich der $\mathrm{Na}$ senhöhle, Dorsalverlagerung des Gaumensegels (Pfeil) als Folge der erschwerten Atmung. b) Transversalschnitt des Kopfes eines anderen Jungrindes mit nasaler Zyste auf Höhe des Unterkiefer-Diastemas. Zyste (Z) liegt im rechten Nasengang und verdrängt das Nasenseptum zur gesunden Seite hin. $\mathrm{T}=$ Tracheotubus.

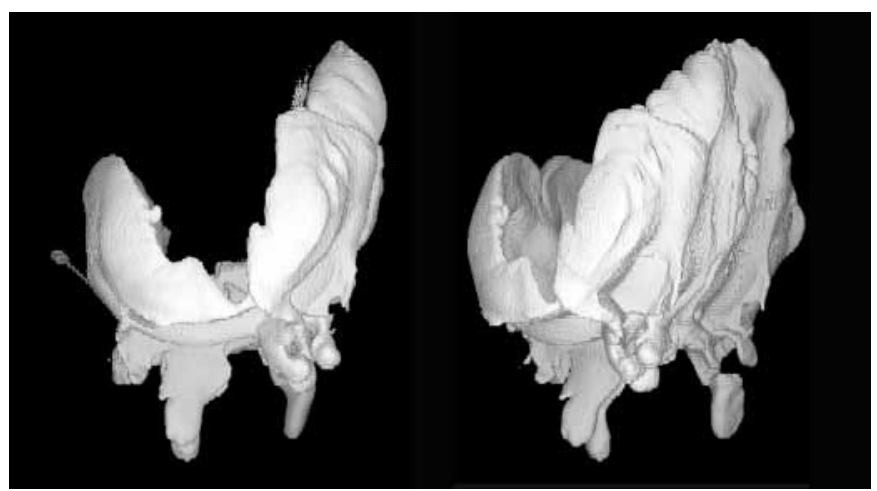

Abb. 5 Dreidimensional wirkende Rekonstruktionen des kontrastmittelgefüllten Fesselgelenks. Die anatomischen Details der Gelenkhöhle sind gut erkennbar und erleichtern dadurch Gelenkpunktion, Arthroskopie oder Arthrotomie.

Fig. 5 Computed tomographic re-constructional images with 3D effect of a contrast medium-filled metacarpophalangeal joint. The anatomical details of the joint cavity are clearly seen and serve to facilitate arthrocentesis, arthroscopy or arthrotomy.

Vor der Allgemeinanästhesie bleibt das Rind für 24-36 Stunden ohne Futter, hat aber bis 6 Stunden vor der Narkose freien Zugang zu Trinkwasser. Dadurch wird eine Pansentympanie vermindert, das Risiko der Regurgitation verringert und die ventilatorische Effizienz (vor allem bei Untersuchung in Rückenlage) verbessert (3).

Zur Prämedikation erfolgt eine Injektion von Xylazin in der Dosierung von $0,1-0,2 \mathrm{mg} / \mathrm{kg}$ KM intramuskulär (13), damit das kardiovaskuläre System eine längere Adaptationszeit hat als bei intra-

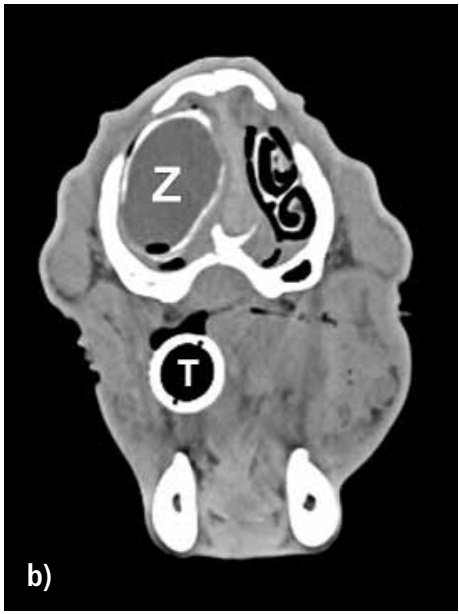

Fig. 4 a) Laterolateral radiograph of a conchonasal cyst in a heifer with inspiratory and expiratory stertor. A mass with soft tissue density (Z) and surrounded by a dense bony capsule is visible in the nasal passage. Laboured breathing has resulted in dorsal displacement of the soft palate (arrow); b) Transverse computed tomographic image of the head of another heifer showing a nasal cyst at the level of the mandibular diastema. The cyst (Z) is located in the right nasal passage and has displaced the nasal septum toward the healthy left nasal passage. $\mathrm{T}=$ endotracheal tube.

venöser Applikation (9). Rinder im letzten Trächtigkeitsdrittel erhalten vor Applikation eines $\alpha_{2}$-Agonisten stets ein Uterusrelaxans (200 mg Isoxuprin i. m.).Zur Prophylaxe allfälliger Myopathien wird zusätzlich präemptiv ein nichtsteroidales Antiphlogistikum verabreicht (z. B. Ketoprofen, $3 \mathrm{mg} / \mathrm{kg} \mathrm{KM}$ ). Die Anästhesie wird 15-20 Minuten nach Gabe von Xylazin mit 2-3 mg/kg KM Ketamin intravenös eingeleitet, das Rind anschließend auf den Untersuchungstisch gehoben und daran fixiert ( Abb. 1). Die Allgemeinanästhesie erfolgt nach endotrachealer Intubation durch Inhalation von Isofluran gelöst in Sauerstoff und Luft. Die inspiratorische Isoflurankonzentration wird für jedes Tier individuell der Anästhesietiefe angepasst. Zur Unterstützung des kardiovaskulären Systems erhält der Patient eine Dauertropfinfusion kristalloider Lösungen. Das Routine-Monitoring umfasst kontinuierliche Elektrokardiographie, Blutdruckmessung (nicht invasiv), Pulsoxymetrie und Kapnographie sowie die Prüfung klinische Parameter (Lidreflex, Bulbusposition, kapilläre Füllungszeit, Schleimhautfarbe). Nach Beendigung der Computertomographie werden die Tiere mit dem Kran auf eine Palette gehoben und in eine Aufwachbox transportiert. Patienten mit wiedererlangtem Bewusstsein und Reflexen werden in Brustlage verbracht und bei deutlich vorhandenem Schluckreflex extubiert.

Eine Kontraststudie, die dem Prinzip der Kontrastmitteldarstellung in der Radiologie folgt, verlängert die Allgemeinanästhesie. Die Darstellung wird üblicherweise mit einem jodhaltigen Kontrastmittel (600-800 mg/kg KM) durchgeführt (20, 21). Zu Darstellung von Gelenken kann das Kontrastmittel im Verhältnis 1:3 mit physiologischer Kochsalzlösung verdünnt werden. Die Zulassungsbeschränkungen für Tiere, die der Lebensmittelgewinnung dienen, sind dabei zu beachten. 


\section{Anwendungen am Kopf}

Indikationen für eine Computertomographie ergeben sich beim Rind am häufigsten bei den Erkrankungen des Kopfes(10, 11, 19, 20). Diese zeigen sich oftmals (z. B. Zahnerkrankungen oder Aktinomykose) in Form von Umfangsvermehrungen. Wenngleich diese durch klinische Untersuchung einfach zu lokalisieren sind, fällt es wegen der komplexen Anatomie der Gewebe des Kopfes meist nicht leicht, das genaue Ausmaß und die involvierten Strukturen zu beschreiben. Die Ultraschalluntersuchung liefert wertvolle Informationen, kann aber Knochenstrukturen oder gasgefüllte Hohlräume nicht durchdringen. Bei der Röntgenuntersuchung ergeben sich oft Interpretationschwierigkeiten, weil sich die Strukturen überlagern und die unterschiedlichen Gewebe keinen guten Kontrast ergeben (29). Computertomographisch lassen sich wichtige Einzelheiten in der Tiefe der Gewebeschichten ( $>$ Abb. 3) darstellen, die vor allem für eine operative Therapie entscheidend sind $(19,38)$. In der Diagnostik der Otitis media beim Kalb hat die Computertomographie für die Darstellung der Bullae tympanicae einen hohen Stellenwert (38).

Im eigenen Patientengut wurde die Computertomographie bisher vorwiegend bei der diagnostischen Abklärung von Erkrankungen der Zähne, der Nasenhöhlen und der Nasennebenhöhlen eingesetzt und ermöglichte eine bessere Differenzierung von Erkrankungen des Unter- und Oberkieferknochens sowie der Zähne. Auch bisher beim Rind wenig bekannte Erkrankungen, wie nasale Zysten oder Sinuszysten, konnten mithilfe der Computertomographie eindeutig diagnostiziert werden. Dabei gelang es ausgezeichnet, die Größe und genaue Lage von Sinuszysten ( $>$ Abb. 4) zu be- stimmen, was deren operative Entfernung erst ermöglichte. Weiterhin ließen sich Tumore (19) sowie die knöchernen Veränderungen bei Stirnhöhlenentzündungen darstellen. Die Kenntnis der Lokalisation und Morphologie eines pathologischen Prozesses erleichterte die Therapieplanung und -durchführung erheblich.

Nach Angaben in der Literatur findet die Computertomographie auch bei neurologischen Erkrankungen (20,37) oder Missbildungen (7) Anwendung, um eine Diagnose intra vitam stellen zu können. So ergaben sich bei 18 Rindern mit neurologischen Erkrankungen folgende Diagnosen: Hydrozephalus $(n=3)$, intrakranielle Arachnoidalzysten ( $n=3)$, Otitis media $(n=5)$ sowie zerebraler Abszess, Meningoenzephalozele, Porenzephalie, Missbildung (Bizephalus) und Rückenmarkabriss (je $n=1$ ) (20). Erkrankungen, die in dieser Untersuchung nicht computertomographisch erfasst werden konnten, waren Kleinhirnherniation, Hirnstammabszess, Hypoplasie des Chiasma opticum, Polyenzephalomalazie und Sklerosierung der weißen Substanz (20). Die Darstellung dieser Erkrankungen gelang vermutlich deshalb nicht, weil die Aussagekraft computertomographischer Bilder von Weichteilstrukturen limitiert ist und Artefakte vorzugsweise in bestimmten Regionen, beispielweise im Bereich der Medulla oblongata, entstehen (15).

\section{Anwendungen an Wirbelsäule, Thorax und Abdomen}

Bei erwachsenen Rindern ist es gerade noch möglich, neben dem Kopf und den Gliedmaßen die Halswirbelsäule zu untersuchen,
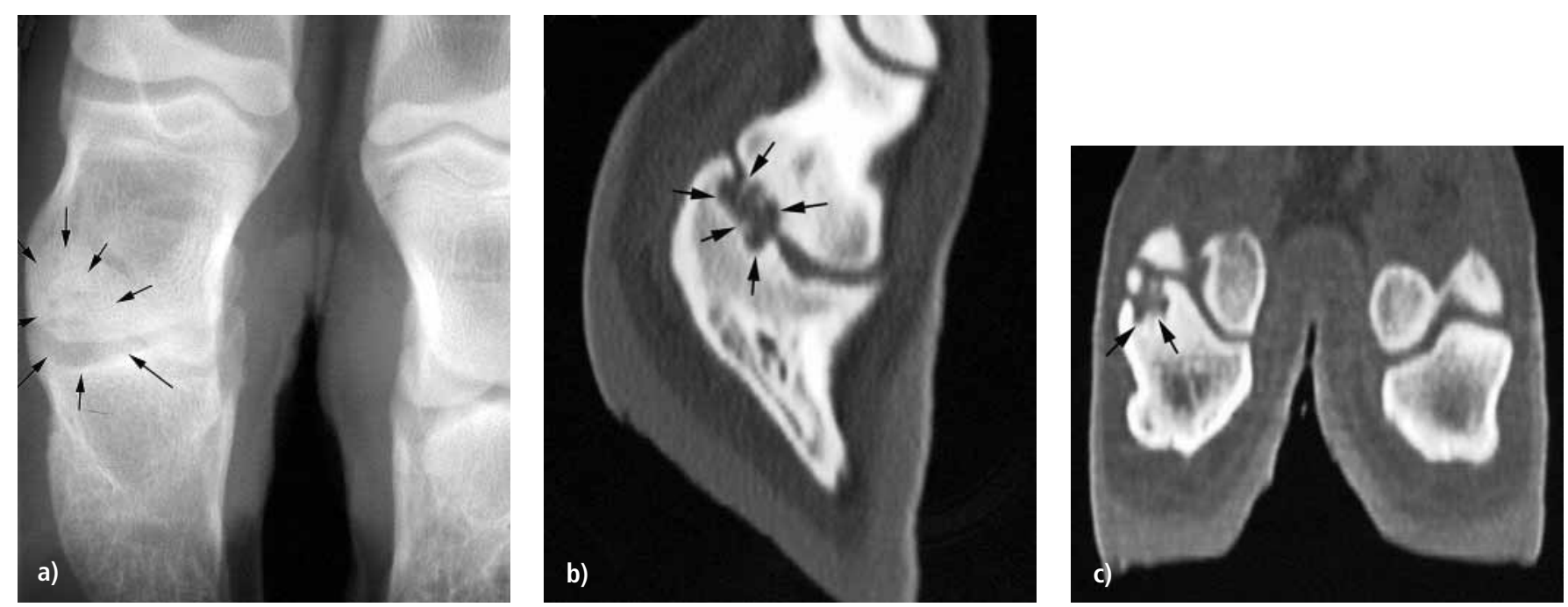

Abb. 6 Schwarzbuntes Rind, 12 Monate, chronische Lahmheit der linken Beckengliedmaße. Schmerzhaftigkeit durch Dreipunktanästhesie auf die Innenklaue lokalisiert. a) Im Röntgenbild auffällige fleckige Röntgendichte im Bereich des Tuberculum flexorium (Pfeile). b, c) Verdeutlichung der Veränderungen durch die computertomographische Untersuchung. Arthrotisch-ankylosierende Veränderung des Klauengelenks unbekannter Ätiologie.

Fig. 6 Twelve-month-old Holstein heifer with chronic lameness of the left hind foot. Pain was localised to the inner claw using nerve blocks. a) Radiograph shows markedly radiodense flecks in the region of the tuberculum flexorium (arrows). b, c) The changes are seen more clearly in the computed tomographic images. The aetiology of the arthrotic ankylosing lesions in the distal interphalangeal joint was unknown. 
während bei Kälbern die gesamte Wirbelsäule untersucht werden kann. Zwar liefert die Röntgenuntersuchung in vielen Fällen ausreichende Befunde für eine Diagnose, aber das Ausmaß der Läsion, beispielsweise angrenzende Abszesse und Einengungen des Wirbelkanals, lassen sich nur computertomographisch genauer darstellen (12).

Infolge der kurzen Aufnahmezeiten von Computertomographen der neuesten Generation (die Darstellung des Kopfes eines erwachsenen Rindes beansprucht weniger als eine Minute Zeit) gelingt es inzwischen auch, Thorax und Abdomen bei kleinen Wiederkäuern und Kälbern abzubilden (5, 6, 22, 23). Der inhärente hohe Weichteilkontrast der Lunge erlaubt eine komplett überlagerungsfreie Darstellung dieses Organs. Die Herzaktion stellt eine Quelle für Bewegungsunschärfe dar. Kurze Aufnahmezeiten sind auch notwendig, um die Entstehung von Respirationsartefakten zu minimieren, die die Beurteilung insbesondere der kaudalen Lungen- und der kranialen Abdomenanteile (Leber) zum Teil deutlich beeinträchtigen. Computertomographische Studien über Thorax und Abdomen werden allerdings als vergleichende anatomische Untersuchungen durchgeführt, sodass bisher wenige Dokumentationen von pathologischen Befunde vorliegen. Weitere Einsatzgebiete der Computertomographie sind die experimentelle Untersuchung des Geburtsvorgangs beim Kalb (Vermessung der Beckengröße von Kühen und der Körperquerschnitte ihrer Kälber [2, 35]), und die Darstellung der postnatalen Lungenentwicklung bei gesunden Kälbern (22).
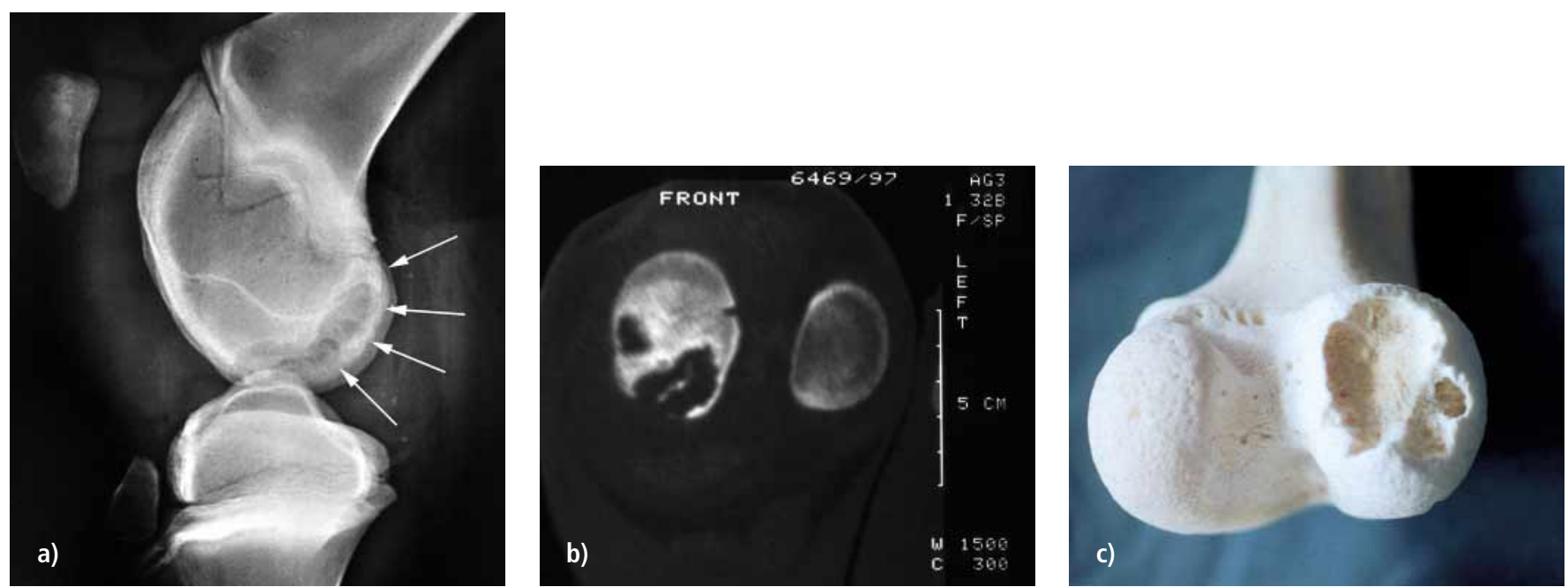

Abb. 7 Fleckvieh-Kalb, 3 Wochen, Lahmheit $4 / 5$ an der rechten Beckengliedmaße im Zusammenhang mit einer septischen Arthritis des Kniegelenks. a) Röntgenbild im mediolateralen Strahlengang: vermehrte Gelenkfüllung, Patella abgehoben, große, unregelmäßige Verschattung im Bereich der Os-femoris-Kondylen. b) Im computertomographischen Bild massive Knochenläsion/-auflösung und zusätzlicher Hohlraum im lateralen Kondylus. c) Entsprechendes Präparat.

\section{Anwendungen an den Gliedmaßen}

Bisher wurden vergleichende anatomische Dissertationen über die Rinderzehe und das Sprunggelenk des Rindes durchgeführt, die einige Bildbeispiele von Erkrankungen beinhalten $(31,33)$. Im Bereich der Gliedmaßen können die Weichteile besser beurteilt werden als mit der konventionellen Radiographie. Auch mögliche Läsionen wie Fissuren lassen sich frühzeitig und weitaus besser in ihrem Verlauf darstellen.

Kontrastdarstellungen von Sehnenscheiden und Gelenken sind bei der Röntgenuntersuchung und der Computertomographie sehr hilfreich. Die Computertomographie erlaubt zusätzlich dreidimensionale Rekonstruktionen (27). Wenn der Computer diese durch die alleinige Auswahl der dichtesten Substanz, des Kontrastmittels, berechnet, entstehen dreidimensionale Rekonstruktionen, die anschaulich sind wie anatomische Ausgusspräparate ( $\triangle$ Abb. 5). Bei Verwendung von Kontrastmittel niedriger Viskosität ist mit der Computertomographie sogar meist die bessere Detaildarstellung möglich, weil ein solches Kontrastmittel in alle Ausbuchtungen des Gelenksacks vordringen und das Gelenk komplett ausfüllen kann (26).

Obwohl sich die Lahmheitsursachen an den Klauen und Gliedsuchung oft einfach diagnostizieren lassen, gibt es Fälle, in denen ohne die Computertomographie keine korrekte Diagnose gestellt werden kann (>Abb. 6). Weiterhin sind insbesondere arthrotische oder durch septische Entzündungen bedingte Veränderungen an Gelenken $(25,39)$, bei denen es zur Überlagerung der beteiligten Knochen und Knochenzubildungen kommt, röntgenologisch nicht immer ausreichend gut darstellbar. Die Computertomographie kann dagemaßen des Rindes mithilfe von Ultraschall- und Röntgenunter- 


\section{Fazit für die Praxis}

Die Computertomographie bietet die Möglichkeit, beim erwachsenen Rind Strukturen an Kopf und Gliedmaßen, bei Kälbern den gesamten Körper überlagerungsfrei und mit hervorragender Kontrastauflösung darzustellen. Sie kann daher beim Nutztier als diagnostisches Verfahren angesehen werden, das als Entscheidungshilfe für schwierige Eingriffe fungieren kann. Nicht zuletzt lassen sich dadurch kostspielige erfolglose Therapieversuche vermeiden. Der Routineanwendung beim Nutztier stehen die Kosten für die Allgemeinanästhesie und für die Untersuchung selbst entgegen. Computertomographen der neuesten Generation verkürzen die Aufnahmedauer stark, sodass künftig eine Kurznarkose für die Untersuchung ausreichen dürfte.

gen das Gelenkinnere und die Knochengrenzen überlagerungsfrei abbilden ( $>$ Abb. 7) und erlaubt eine bessere Diagnosestellung und gegebenenfalls Therapieplanung. Sie dient somit auch dazu, aufwendige erfolglose Behandlungen infolge unzureichender Kenntnis der vorliegenden Veränderungen zu vermeiden.

\section{Interessenkonflikt}

Die Autoren bestätigen, dass kein Interessenkonflikt besteht.

\section{Literatur}

1. Barbee D, Allen J, Grant B, Riggs M, Crawley G, Sande R. Detection by computed tomography of occult osteochondral defects in the fetlock of a horse. Equine Vet J 1987; 19: 556-558.

2. Becker M, Heun C, Tsousis G, Bollwein H. Application of computed tomography for the evaluation of obstetrically relevant measurements in German Holstein-Friesian calves. Theriogenology 2011; 75: 1052-1056.

3. Blaze CA, LeBlanc PH, Robinson NE. Effect of withholding feed on ventilation and the incidence of regurgitation during halothane anesthesia of adult cattle. Am J Vet Res1988; 49 (12): 2126-2129.

4. Böttcher P, Maierl J, Schiemann T, Glaser C, Weller R, Hoehne KH, Reiser M, Liebich HG. The visible animal project: a three-dimensional, digital database for high quality three-dimensional reconstructions. Vet Radiol Ultrasound 1999; 40: 611-616.

5. Braun U, Irmer M, Augsburger H, Jud R, Ohlerth S. Computed tomography of the abdomen in Saanen goats: I. Reticulum, rumen and omasum. Schweiz Arch Tierheilk 2011 (im Druck).

6. Braun U, Irmer M, Augsburger H, Müller U, Jud S, Ohlerth S. Computed tomography of the abdomen in Saanen goats: II. Liver, spleen, abomasum and intestine. Schweiz Arch Tierheilk 2011 (im Druck).

7. Buck B. Untersuchungen zu kongenitalen Anomalien beim Rind. Diss med vet, Tierärztliche Hochschule Hannover 2008.

8. El-Khodery S, Yamada K, Aoki D, Kamio K, Kishimoto M, Shimizu J, Kobayashi $Y$, Ishii $M$, Inokuma $\mathrm{H}$, Yamauchi S, Matsui T. Brain abscess in a Japanese black calf: utility of computed tomography (CT). J Vet Med Sci 2008; 70: 727-730.

9. England GC, Clarke KW. Alpha 2 adrenoceptor agonists in the horse - a review. Br Vet J 1996; 152: 641-657.

10. Finnen A, Blond L, Francoz D, Parent J. Comparison of computed tomography and routine radiography of the tympanic bullae in the diagnosis of otitis media in the calf. J Vet Intern Med 2011; 25: 143-147.

11. Frame EM, Gufler H, Henninger W, Gasteiner J. Diagnosis of atrophic rhinitis in a Simmental heifer by computed tomography. Vet Rec 2000; 146 : 558-560.
12. Frederick JD, Mackay RJ, Winter MD, Castleman WL. Vertebral osteomyelitis with abscessation in a Holstein calf. Vet Rec 2009; 164: 723-724.

13. Greene SA. Protocols for anesthesia of cattle. Vet Clin North Am Food Anim Pract 2003; 19: 679-693.

14. Hathcock JT, Stickle RL. Principles and concepts of computed tomography. Vet Clin North Am Small Anim Pract 1993; 23: 399-415.

15. Hecht S, Morandi F. Weiterführende Bildgebende Verfahren. In: Röntgendiagnostik in der Kleintierpraxis. Hecht S, Hrsg. Stuttgart: Schattauer 2008; 39-52.

16. Hounsfield GN. Computerized transverse axial scanning (tomography). 1. Description of system. Br J Radiol 1973; 46: 1016-1022.

17. Hounsfield GN. Computed medical imaging. Science 1980; 210: 22-28.

18. Kaser-Hotz B, Sartoretti-Schefer S, Weiss R. Computed tomography and magnetic resonance imaging of the normal equine carpus. Vet Rad Ultrasound 1994; 35: 457-461.

19. Keller A, Nitzl D, Hauser B, Wehbrink D, Muggli E, Nuss K. Diagnostik und operative Behandlung eines ameloblastischen Fibrodentinoms bei einer Kuh. Tierärztl Prax 2007; 35 (G): 255-261.

20. Lee K, Yamada K, Tsuneda R, Kishimoto M, Shimizu J, Kobayashi Y, Furuoka H, Matsui T, Sasaki N, Ishii M, Inokuma H, Iwasaki T, Miyake Y. Clinical experience of using multidetector-row CT for the diagnosis of disorders in cattle. Vet Rec 2009; 165: 559-562.

21. Lee KJ, Kishimoto M, Shimizu J, Kobayashi Y, Matsumoto K, Sasaki N, Ishii M, Inokuma H, Iwasaki T, Miyake Y, Yamada K. Use of contrast-enhanced CT in the diagnosis of abscesses in cattle. J Vet Med Sci; 73: 113-115.

22. Linke B. Computertomographische Untersuchungen zur Erfassung der Lungenfunktion bei vitalen Kälbern in der frühen postnatalen Phase. Diss med vet, Fachbereich Veterinärmedizin, Universität Gießen 2009.

23. Lubbers BV, Apley MD, Coetzee JF, Mosier DA, Biller DS, Mason DE, HenaoGuerrero PN. Use of computed tomography to evaluate pathologic changes in the lungs of calves with experimentally induced respiratory tract disease. Am J Vet Res 2007; 68: 1259-1264.

24. Morandi F, Mattoon JS, Lakritz J, Turk JR, Wisner ER. Correlation of helical and incremental high-resolution thin-section computed tomographic imaging with histomorphometric quantitative evaluation of lungs in dogs. Am J Vet Res 2003; 64: 935-944.

25. Nuss K, Roth M, Schäffer E. Deformierende idiopathische Ankylose der Klauengelenke beim Jungrind. Tierärztl Prax 1994; 22: 312-318.

26. Nuss K. Stadienorientierte Sequenztherapie der septischen Monarthritis beim Rind. Habilitationsschrift, Tierärztliche Fakultät, Universität München 2000.

27. Nuss K, Hecht S, Maierl J, Böttcher P. Contrast radiography and 3D reconstruction of bovine limb joint cavities. In: Proc 10th Annual ESVOT Congress, Munich, Germany 2000; 129.

28. O'Brien RT, Biller DS. Clinical applications of radiography and ancillary imaging. Vet Clin North Am Food Anim Pract 1996; 12: 263-275.

29. Pease A. The Equine Head. In: Textbook of Veterinary Diagnostic Radiology, 5th ed. Thrall D, ed. St. Louis: Saunders Elsevier 2007; 160-178.

30. Raij A, Sardari K, Mohammadi H. Normal cross-sectional anatomy of the bovine digit: comparison of computed tomography and limb anatomy. Anat Hist Embryol 2008; 37: 188-191.

31. Schwarze I. Computertomographische Untersuchungen am Tarsus des Rindes. Diss med vet, Tierärztliche Fakultät, Universität München 1998.

32. Tidwell A. Principles of computed tomography and magnetic resonance imaging. In: Textbook of Veterinary Diagnostic Radiology, 5th ed. Thrall D, ed. St. Louis: Saunders Elsevier 2007; 50-77.

33. Tiefenthaler I. Computertomographische Untersuchung der Rinderzehe. Diss med vet, Tierärztliche Fakultät, Universität München 1997.

34. Tietje S. Zur Bedeutung der Computertomographie beim Pferd (243 Fälle). Prakt Tierarzt 1997; 78: 35-43.

35. Tsousis G, Heun C, Becker M, Bollwein H. Application of computed tomography for the evaluation of obstetrically relevant pelvic parameters in German Holstein-Friesian cows. Theriogenology 2011; 73: 309-315.

36. Tsuka T, Taura Y. Abscess of bovine brain stem diagnosed by contrast MRI examinations. J Vet Med Sci 1999; 61: 425-427. 
37. Tsuka T, Taura Y, Okamura S, Tamura H, Okamoto Y, Okamura Y, Minami S. Imaging diagnosis - polioencephalomalacia in a calf. Vet Radiol Ultrasound 2008; 49: 149-151.

38. Van Biervliet J, Perkins GA, Woodie B, Pelligrini-Massini A, Divers TJ, de Lahunta A. Clinical signs, computed tomographic imaging, and management of chronic otitis media/interna in dairy calves. J Vet Intern Med 2004; 18: 907-910.
39. Willemen M, Dik K. Bipartite distal sesamoid bones in a Holstein-Friesian calf. Vet Rec 1995; 137: 42-43.

40. Wuersch K, Bischoff M, Thoma R, Matter MS. Nasal osteoma in a dairy cow: a combined clinical, imaging and histopathological approach to diagnosis. J Comp Pathol 2009; 141: 204-207.

41. Zani DD, Romano L, Scandella M, Rondena M, Riccaboni P, Morandi N, Lombardo R, Di Giancamillo M, Belloli AG, Pravettoni D. Spinal epidural abscess in two calves. Vet Surg 2008; 37: 801-808. 